\title{
Examining the Relationship between Spatial Patterns of Household Deprivation and Some Socioeconomic Indexes in Iraq-2004
}

\author{
Faisal G. Khamis ${ }^{1} \&$ Ghaleb A. El-Refae ${ }^{2}$ \\ ${ }^{1}$ Banking \& Finance Department, Faculty of Economics and Administrative Sciences, AL-Zaytoonah University \\ of Jordan, Amman, Jordan \\ ${ }^{2}$ Banking \& Finance Department, Al Ain University of Science and Technology, Al Ain, U.A.E. \\ Correspondence: Faisal G. Khamis, Banking \& Finance Department, Faculty of Economics and Administrative \\ Sciences, AL-Zaytoonah University of Jordan, Amman, Jordan. E-mail: faisal_alshamari@yahoo.com
}

Received: May 25, 2012 Accepted: June 29, 2012 Online Published: September 20, 2012

doi:10.5539/ass.v8n12p95 URL: http://dx.doi.org/10.5539/ass.v8n12p95

\begin{abstract}
Regions, independent of their geographic level of aggregation, are known to be interrelated partly due to their relative locations. Similar economic performance among regions can be attributed to proximity. Consequently, a proper understanding and accounting of spatial liaisons are needed in order to effectively forecast regional economic variables. In this research household deprivation (HD) is viewed from the perspective of living standards index (LSI). Historically, governorates in Iraq suffered inequalities of living standards for many reasons, such as government's focus on the heart of the city, specifically Baghdad, Basra, and Nineveh. Question is raised whether the spatial pattern of HD is existed in Iraq? If so, can household economic status (HES) and education indexes explain this pattern?

The objective is to investigate spatial structure of LSI and its spatial correlation to spatial structure of each of HES and education. This investigation will provide implications for policy makers, finding local clusters and showing visual picture for each of LSI, HES and education.

The study utilizes a cross-sectional census data collected in 2004 for 18 governorates. Mapping is used as the first step to conduct visual inspection for LSI using quartiles. Several spatial econometric techniques are available in the literature, which deal with the spatial autocorrelation in geographically referenced data. Two statistics of spatial autocorrelation, based on sharing boundary neighbours, known as global and local Moran's $I$, are carried out. Wartenberg's measure is used to detect bivariate spatial correlation.

The hypothesis of spatial clustering for LSI was confirmed by a positive global Moran's $I$ of .34 with $\mathrm{p}=.002$ and permutation $\mathrm{p}=.006$, while for education was not confirmed by a negative global Moran's $I$ of -.16 with $\mathrm{p}=.451$ and for HES was confirmed by a positive global Moran's $I$ of .28 with $\mathrm{p}=.010$. Bivariate spatial correlation between LSI and education wasn't found significant .09 with $\mathrm{p}=.165$ and between LSI and HES was found significant .31 with $\mathrm{p}=.002$.

In conclusion, based on visual inspection of mapping global clustering was found in LSI on the northern and southern parts. It was confirmed by the significant global Moran's I statistic. Out of 18 governorates; eight, seven, and one were found as local clusters in LSI, HES, and education respectively based on local Moran's $I_{i}$. The bivariate spatial correlation between LSI and education was not found significant while between LSI and HES was found significant.
\end{abstract}

Keywords: spatial autocorrelation, deprivation, mapping, Moran statistics, Iraq's governorates, socioeconomic indexes

\section{Introduction}

Spatial statistics relates to the analysis of the spatial aspect of data sets. All data have, implicitly or explicitly, a spatial component. The reasons why spatial statistics are of import for many areas are threefold: (i) Data that are spatially close, are usually more similar than those that are further apart. Hence, there is spatial (and temporal) dependence for most data sets. (ii) Spatial models to explain and make inference about data structures have important implications in many fields. (iii) The literature on spatial statistics is substantial, but there still is a lot to uncover and many questions to answer. 
Deprivation indicates the condition of poverty perceived as "human poverty," which includes income poverty, as well as its social, human, and capable dimensions. Deprivation is the lack of welfare. It is often understood in terms of material goods and resources but equally applicable to psychological factors. It is relative to the local community, the wider society, or nation to which an individual, family, or group belongs (Gordon \& Spicker, 1999). The two concepts of poverty and deprivation are tightly linked, but there is general agreement that the concept of deprivation covers various conditions, independent of income, and experienced by people who are poor. Whereas, the concept of poverty refers to the lack of income and other resources, that make those conditions inescapable or at least highly likely (Townsend, 1987). Hooghe et al. (2011) investigated the impact of deprivation indicators on crime in Belgian municipalities for the period 2001-06. A spatial regression analysis used by Hooghe et al. demonstrated that unemployment figures have a strong and significant impact on crime rates. In a spatial analysis conducted in Madrid, it was found that mortality increased with deprivation (Pozo et al., 2010).Exploratory spatial data analysis conducted by Sridharan, Koschinsky, and Walker (2011) revealed concentrations of high standardized mortality ratios and deprivation (hotspots) in the West of Scotland and concentrations of low values (coldspots) for both variables in the rest of the country.

In recent years, a growing interest has been seen in examining the existence of spatial autocorrelation of deprivation and its spatial relationship to several indicators such as unemployment, education, etc. across governorates in Iraq. Twenty-five years ago, Iraq was a country having the best living standard comparing to its neighbours. Recently, it has fallen due to some indicators. In some cases, it has fallen far behind. The growing income inequality has contributed significantly to the deterioration of deprivation in Iraq. By the beginning of the 1980 s, other factors contributed to the rise in disparities among the governorates, including the geographic location of the governorate in terms of its proximity to battlefields, and the social composition of the governorates' population, especially in north and south. In other governorates, disparities stemmed according to political and tribal origins.

Deprivation was studied in many countries using different statistical measures. Hulme, Shepherd, and Spray (2005) stated that Between 300 and 420 million people were trapped in chronic poverty. They experience deprivation over many years, often over their entire lives, and commonly pass poverty on to their children. Many chronically poor people die prematurely due to health problems that are easily preventable. For them, poverty is not simply having a low income: it is about multidimensional deprivation- hunger, malnutrition, dirty drinking water, illiteracy, having no access to health services, social isolation, and exploitation. Such deprivation and suffering exist in a world that has the knowledge and resources to eradicate it. Patterning of deprived neighbourhoods in Glasgow in Scotland may in part be an explanation for differences between health outcomes in Glasgow and similar deprived postindustrial cities (Livingston et al., 2011). Material deprivation combined with isolation creates peripheral poverty that can be found in marginal areas. Overcrowding poverty is a result of material deprivation caused by population pressure and limited resources (Henninger, 1998). Sven, Ursula, and Razum (2010) highlighted the difference regional and in particular neighbourhood deprivation make to the physical health of individuals in Germany. Lamb et al. (2010) concluded that area-level deprivation appears to have a significant association with the density of physical activity facilities and although overall no clear pattern was observed, affluent areas had fewer publicly owned facilities than more deprived areas, but a greater number of privately owned facilities. There is greater variation in death rates and socio-demographic characteristics among the most deprived constituencies in Britain. The socio-demographic factors that are most strongly correlated with death rates among the most deprived places differ from areas of all deprivation levels and include population density, ethnicity and migration (Tunstall et al., 2011).

Most of the above studies have shown that deprived persons have an increased risk of death. Over half the children in the World suffer from at least one severe deprivation of basic human need; $30 \%$ of them are absolutely poor (Gordon et al., 2007). To understand the linkages between socioeconomic variables, investigations should focus on features of the areas rather than on the compositional characteristics of residents of the area, which cannot fully describe the social environment in which people live (Macintyre, Maciver \& Sooman, 1993). So, the aim of the research is to study spatial autocorrelation and geographical mapping regarding household deprivation and its spatial relationship to some socioeconomic indicators. Spatial autocorrelation is the term used for the interdependence of the values of a variable over space. However, it was argued that lattice data are spatially correlated, where exploratory spatial data analysis (ESDA) was used using lattice data. The ESDA quantifies the spatial pattern in order to increase the analyst's knowledge of the spatial system. As well as mapping plays an important role in monitoring deprived people. Maps can reveal spatial patterns that is neither recognized previously nor suspected from the examination of statistics table. It reveals high risk communities or problem areas (Lawson \& Williams, 2001). The purpose of spatial analysis is to 
identify pattern in geographical data attempts to explain this pattern. Findings are expected to enhance deprivation monitoring and policy interventions across governorates of Iraq.

In this research some hypotheses are tested. Are household deprivation and some socioeconomic indicators having global clustering across Iraq's governorates? Are there local clusters? If so, how many clusters are there and where are they located? Then is clustering in household deprivation can be explained by clustering in socioeconomic indicators? Governorates are tightly linked by migration, commuting, and inter-governorate trade. These types of spatial interaction are exposed to the frictional effects of distance, possibly causing spatial dependence of governorate labour market conditions. Some of socioeconomic indicators such as education basically offer greater chance of getting a job which in turn is considered a major source of income that avoids falling in deprivation. The HES including financial status, employment, etc. is also considered a major determinant leads to deprivation.

Research relevance stems from a statement states that reducing deprivation inequalities is not a primary objective but emergent prosperity. Importance of research objective emanates the study conducted in Jordan by Amerah (1993), stated that health was affected negatively by deprivation. However, it is very necessary for policy makers to know in which area the problem of deprivation inequality is existed and what socioeconomic indicators can spatially relate to this problem? Also, to authors' knowledge, no studies used spatial analysis techniques and geographical mapping in studying inequality in HD in Iraq.

Importance of mapping was stated by Koch (2005): why make the map if detailed statistical tables carry the same results? Perhaps the most important reason for studying spatial statistics is not only interested in answering the "how much" question, but the "how much is where" question (Schabenberger \& Gotway, 2005). In light of these: (1) the existence of spatial global clustering, (2) spatial local clusters for each of LSI, HES, and education are investigated, (3) mapping is applied for each of LSI, HES, and education and for their local Moran's $I_{i}$ values, and (4) bivariate spatial correlation between LSI and each of HES, and education are examined based on Wartenberg's (1985) statistic. The study design was a cross-sectional analysis in a census survey conducted in Iraq in 2004. Findings make a significant contribution by moving beyond the investigation of a single socioeconomic resource. However, findings push us to more fully consider where and why LSI, HES, and education matter.

The paper is structured as follows: Section one reviews the literature relating to HD and socioeconomic disparities generally in several countries and particularly in Iraq which is the author interest to study. Materials and methods including data details and statistical analysis are presented in second Section. Third section shows the results with some details. Discussion is explained in fourth Section. Final section is closed with several conclusions.

\section{Materials and Methods}

\subsection{Data}

Data were collected from the ministry of planning and development cooperation (2006), based on census conducted in Iraq in 2004. The LSI (a composite index) was investigated using factor analysis. The population size in each of eighteen governorates is shown in Table 3. However, the population size in these governorates is not equal, meaning that the rate of deprivation does not express the absolute size of the problem. The LSI is an important indicator of the level of deprivation within the geographic area forming the governorate. It is an indicator that should be relied upon to determine intervention priorities at the regional level.

The LSI covered six fields: Education, Health, Infrastructure, Housing, Housing environment, and Household economic status (HES). Education field includes five indicators: Enrollment, attained educational level of adults, time needed to reach primary school, time needed to reach secondary school, and satisfaction level of school. Health field includes seven indicators: Number of household members suffering physical chronic diseases or health problems, underweight of children (weight to age), stunting (height to age), advice on health care during last pregnancy, time needed to reach a public hospital, time needed to reach a primary health care center/doctor, and satisfaction level with health services. Infrastructure field includes seven indicators: Main source of drinking water, availability of drinking water, satisfaction level with the quality of water, availability of a source of electricity, stability of electricity supply from the public network, sanitation facilities, and solid waste disposal method. Housing field includes five indicators: Construction material of ceilings, per capita share of rooms, kind of fuel used in heating, satisfaction with overall quality of housing (space, privacy, cost, noise, and environment inside the house), and the number of pollutants inside the house (three of which are: feces inside the house or its inside yard, still water, and waste water).Housing environment field includes nine indicators: Satisfaction level with transportation and traffic, the quality of road used to reach the house, access to ambulances/fire trucks, 
satisfaction levels with shops/markets, satisfaction level with cleanness and pollution control outside the house, number of pollutants in the dwelling area (five of which are: Garbage, waste water, smoke, damaged military vehicles, weapons stocks), number of undesired sites around the house (eight of which are: River, railway, highway, industrial areas, high density areas, garbage dump, and decline and approach to valley), satisfaction level with safety conditions for children outside the house, and gunfire outside the house. HES field includes nine indicators: Average per capita income, possibility of securing one hundred thousand dinar (USD70) in one week, satisfaction level with work opportunities and availability of work, work status of household members, economic dependency rate (size of household divided by number of employed members), number of durable goods (out of sixteen commodities), assets ownership (house, car, and income from property), capability to satisfy specific needs (six of which are: Maintaining a warm house in winter and a cool house in summer, one weeks' vacation outside the house, changing the furniture, buying clothes, and eating fish and meat), and household assessment of its overall economic status.

The scoring of the indicators of the LSI ranged between $(0=$ the lowest level of satisfaction, determined by the applied questionnaire and $2=$ the highest level of satisfaction of needs). The threshold for determining deprivation was one, whereby a deprived person or household scores less than one for any given indicator, topic or for the overall LSI was considered to be living in depravity.

Poverty in Iraq is not only linked to economic resources, but also to the transitional period, the deterioration of services and institutions, and the instability suffered by Iraqi society. Thus, viewing poverty from the perspective of living standards and human deprivation within a group of areas (health, education, housing, services, safety, economic resources, etc.) is both more responsive and more suitable.

Deprived households are shown to be strongly characterized by a high number of children. $43 \%$ of individuals under 15 years of age live in households with low living standards, compared to $39 \%$ in households with medium living standards and $34 \%$ for high-level standards. In contrast, the opposite trend is noticed for individuals aged 34-64. Thus, deprived households have a higher percent of members not engaged in economic activities and fewer members aged 34-64.

According to LSI, $31 \%$ of households and $34 \%$ of individuals suffered from deprivation in 2004. These are households and individuals found in the low living standards category (totaling the low and very low living standards category). Out of this percent, $5 \%$ of the households and $6 \%$ of the individuals were living in very low living standards; representing the population living in extreme poverty. Households were deprived with $55 \%$ had low standard of living, $27 \%$ had a medium standard of living and $18 \%$ had high living standards. The high deprivation results originated from the impact of state policies led to decreasing the real household income. In Iraq, $55 \%$ and $32 \%$ of households were deprived in the fields of HES and education respectively. According to LSI, AL-Muthana governorate accounted for the highest rate of household deprivation $56.4 \%$. It was followed by the governorates of Babil and AlQadisiyah, as accounted for $55.5 \%$ and $51.6 \%$, respectively. The lowest rate was found in Erbil governorate 15.5\%, followed by the governorates of Kirkuk and Baghdad. This can be explained by the persistent growth of economic activity in most fields. It provides more job opportunities especially for those who have academic qualifications. The overall LSI, education, and HES score for the whole of Iraq were $31.2 \%, 31.8 \%$, and $55.1 \%$ respectively. Descriptive statistics were calculated for LSI, where the mean and standard deviation were found 35.81 and 12.98 respectively; skewness and kurtosis were found 0.19 and -1.30 respectively. The five-number summary of LSI data set consisted of the minimum, maximum and quartiles written in increasing order: $\mathrm{Min}=15.50, \mathrm{Q}_{1}=26.88, \mathrm{Q}_{2}=31.25, \mathrm{Q}_{3}=47.98$ and $\mathrm{Max}=56.40$. From the five-number summary, the variations of the four quarters were found $11.38,4.37,16.73$ and 8.42 respectively, where the $3^{\text {rd }}$. quarter has the greatest variation of all. Baghdad, the capital, was considered the least deprived in terms of overall standards of living and of three fields: education, health, and housing.

\subsection{Analysis}

Data analysis involved six steps. In step 1, the LSI, HES, and education were tested for normal distribution. They were found to follow approximately normal distribution. In step 2, visual inspection based on the quantified gradients for each of LSI, HES, and education using quartiles were conducted. Step 3 included the calculation of global Moran's I-statistic for each of LSI, HES, and education to detect the global clustering and the significance of I-statistic using permutation test was examined. Step 4 involved the calculation of local Moran's $\mathrm{I}_{\mathrm{i}}$ for ith governorate and its p-value using Monte Carlo simulation to detect the local clusters for each of LSI, HES, and education. In step 5, using quartiles, visual inspection for the gradients of local Moran values was inspected based on choropleth mapping. In Step 6, the bivariate spatial correlation between LSI and each of HES and education was examined based on Wartenberg's (1985) measure. 
The LSI was categorized by four intervals. These intervals were used for all maps using darker shades of gray to indicate increasing values of LSI. Such approach enables qualitative evaluation of spatial pattern. In the neighbourhood researches, neighbours may be defined as governorates which border each other or within a certain distance of each other. In this research neighbouring structure was defined as governorates which share a boundary. The second order method (queen pattern) which included both the first-order neighbours (rook pattern) and those diagonally linked (bishop pattern) was used. A neighbourhood system for Iraq's governorates is explained in Figure 1, where the ID neighbours for each governorate are shown.

To construct a choropleth map, data for enumeration governorates are typically grouped into classes and a gray tone was assigned to each class. Although maps allow visual assessment for spatial pattern, they have two important limitations: their interpretation varies from person to person, and there is the possibility that a perceived pattern is actually the result of randomness, and thus not meaningful. For these reasons, it makes sense to compute a numerical measure of spatial pattern, which can be accomplished using spatial autocorrelation.

\subsubsection{Identification of Global Spatial Clustering}

The goal of a global index of spatial autocorrelation is to summarize the degree to which similar observations tend to occur near to each other in geographic space. In this exploratory spatial analysis, the spatial autocorrelation using standard normal deviate (z-value) of Moran's $I$ under normal assumption was tested. Moran's $I$ is a coefficient used to measure the strength of spatial autocorrelation in regional data. The null hypothesis of no spatial autocorrelation or spatially independent versus the alternative of positive spatial autocorrelation is as follows:

$$
\begin{aligned}
& \mathrm{H}_{0} \text { : No clustering exists (no spatial autocorrelation) } \\
& \mathrm{H}_{1} \text { : Clustering exists (positive spatial autocorrelation) }
\end{aligned}
$$

Moran's Iis calculated as follows (Cliff \& Ord, 1981):

$$
I=\frac{N \sum_{i=1}^{N} \sum_{j=1}^{N} w_{i j}\left(x_{i}-\bar{x}\right)\left(x_{j}-\bar{x}\right)}{S_{0} \sum_{i=1}^{N}\left(x_{i}-\bar{x}\right)^{2}} \text { and } S_{0}=\sum_{i=1}^{N} \sum_{j=1}^{N} w_{i j}, i \neq j
$$

where, $\mathrm{N}=18$ is the number of governorates, the $\mathrm{w}_{\mathrm{ij}}$ is a weight denoting the strength of the connection between two governorates $i$ and $j$, otherwise, $w_{i j}=z e r o$, and the $x_{i}$ and $x_{j}$ represents the LSI, HES, or education in ith and jth governorate respectively.

A significant positive value of Moran's I indicates positive spatial autocorrelation, showing the overall pattern for the governorates having a high/low level of LSI similar to their neighbouring governorates. A significant negative value indicates negative spatial autocorrelation, showing the governorates having a high/low level of LSI unlike neighbouring governorates. To test the significance of global Moran's I, $z$-statistic which follows a standard normal distribution was applied. It is calculated as follows (Weeks, 1992):

$$
z=\frac{I-E(I)}{\sqrt{\operatorname{var}(I)}}
$$

Permutation test was applied. A permutation test tells us that a certain pattern in data is or is not likely to have arisen by chance. The observations of each variable as randomly reallocated 1000 times with 1000 of spatial autocorrelations were calculated in each time to test the null hypothesis of randomness. The hypothesis under investigation suggests that there will be a tendency for a certain type of spatial pattern to appear in data, whereas the null hypothesis says that if this pattern is present, then this is a pure chance effect of observations in a random order.

The analysis suggests an evidence of clustering if the result of the global test is found significant; though it doesn't identify the locations of any particular clusters. Besides, clustering that represent global characteristic of LSI, the existence and location of localized spatial LSI clusters are of interest in geographic sociology. Accordingly, local spatial statistic was advocated for identifying and assessing potential clusters.

\subsubsection{Identification of Local Spatial Clusters}

A global index can suggest clustering but cannot identify individual clusters (Waller \& Gotway, 2004). Anselin (1995) proposed the local Moran's Ii statistic to test the local autocorrelation. Local spatial clusters, sometimes referred to as hot spots, may be identified as those locations or sets of contiguous locations for which the local 
Moran's Ii is significant. However, Moran's Ii for ith governorate may be defined by Waller and Gotway (2004) as:

$$
I_{i}=\frac{\left(x_{i}-\bar{x}\right)}{S} \sum_{j=1}^{N}\left(w_{i j} / \sum_{j=1}^{N_{i}} w_{i j}\right) \frac{\left(x_{j}-\bar{x}\right)}{S}, i=1,2, \ldots, 18
$$

Where, analogous to the global Moran's $I$, the $x_{i}$ and $x_{j}$ represents the LSI, HES, or education in ith and $j$ th governorate respectively, $N_{i}=$ number of neighbours for ith governorate, and $S$ is the standard deviation of LSI. It is noteworthy to mention that the number of neighbours for ith governorate were taken into account by the amount: $\left(w_{i j} / \sum_{j=1}^{N_{i}} w_{i j}\right)$, where $w_{i j}$ was measured in the same manner as in Moran's I statistic. Local Moran statistic was used to test the null hypothesis of no clusters.

Cluster could be due to either aggregation of high values, aggregation of low values, or aggregation of moderate values. Thereby, high values of $I_{i}$ suggesting a cluster of similar (but not necessarily large) values across several governorates, and low value of $I_{i}$ suggesting an outlying cluster in a single governorate $i$ (being different from most or all of its neighbours). A positive local Moran value indicates local stability, such as a governorate that has high/low LSI surrounded by governorate that has high/low LSI. A negative local Moran value indicates local instability, such as a governorate having low LSI surrounded by governorate having high LSI or vice versa. However, each governorate's $I_{i}$ value was mapped to provide insight into the location of governorates with comparatively high or low local association with their neighbouring values. In the statistical analysis, all programs were performed using SPLUS8 Software.

\subsubsection{Bivariate Spatial Association}

So far, only univariate spatial correlation is presented. It quantifies the spatial structure of one variable at a time. There is much discussion about what is an appropriate measure for bivariate spatial association. However, spatial dependence or spatial clustering causes losing in the information that each observation carries. When $N$ observations are made on a variable that is spatially dependent (and that dependence is positive so that nearby values tend to be similar).The amount of information carried by the sample is less than the amount of information that would be carried, if the $N$ observations are independent. Due to a certain amount of information carried by each observation is duplicated by other observations in the cluster. A general consequence of this is that the sampling variance of statistics is underestimated. As the level of spatial dependence increases, the underestimation increases. The problem prevails when spatial autocorrelation is present, the variance of the sampling distribution of e.g. Pearson correlation coefficient, which is a function of the number of pairs of observations, is underestimated. Spatial autocorrelation coefficient can be modified to estimate the bivariate spatial correlation between two variables (Wartenberg, 1985):

$$
I_{x y}=\frac{1}{S_{0}} \frac{\sum_{i=1}^{N} \sum_{j=1}^{N} w_{i j}\left(x_{i}-\bar{x}\right)\left(y_{j}-\bar{y}\right)}{\left.\sqrt{\sum_{i=1}^{N}\left(x_{i}-\bar{x}\right)^{2} / N}\right]\left[\sqrt{\sum_{j=1}^{N}\left(y_{j}-\bar{y}\right)^{2} / N}\right]}
$$

Where $x$ and $y$ are the LSI and (education or HES) variables respectively. Although the mathematics is quite straightforward, very few software packages offer the option of computing, $I_{x y}$. Thus, programming was used to find $I_{x y}$. To test the significance of $I_{x y}, \quad z$-statistic was applied: $z=I_{x y} \sqrt{N-1}$, which follows approximately standard normal distribution.

\section{Results}

Factor analysis with a maximum likelihood approach was employed to identify LSI factor. Factor scores of LSI were used as inputs to spatial analysis measures. The results of factor analysis are explained in Table 1. The highest estimated factor loadings of factor 1 were appeared for infrastructure, housing conditions, and housing $(.99, .82$, and .62 respectively).This strong interaction between these variables reflects the effect on LSI. Factor 1 representing (housing, its conditions, and its services) interpreted 53\% of the total variance of studying variables. That's why factor 1 was chosen to represent LSI variable. Because of this, education and HES variables were studied to explain clustering in LSI. These variables were not entered in the structure of LSI factor, where they were entered in construction of factor 2. The highest estimated factor loadings of factor 2 were appeared for 
health, education, and HES $(.98, .70$, and .60 respectively). Factor 2 , representing humanity development, interpreted only $22 \%$ of the total variance. That's why it wasn't used for constructing LSI. A measure of internal coherence such as Cronbach's alpha for LSI (Factor 1) was found accepted .79. Cronbach's alpha is commonly used measure of reliability for a set of two or more construct indicators.

Table 1. Explains the results of factor analysis for six variables

\begin{tabular}{lcc}
\hline & \multicolumn{2}{c}{ Estimated factor loadings } \\
\cline { 2 - 3 } Variable & Factor 1 & Factor 2 \\
\hline HES & .27 & .60 \\
Education & .29 & .70 \\
Housing conditions & .82 & .08 \\
Housing & .62 & .45 \\
Health & -.33 & .98 \\
Infrastructure & .99 & -.19 \\
Factor interpretation & $53 \%$ & $22 \%$
\end{tabular}

Figure 1 shows the study area explaining all governorates with their identification numbers (ID). Figures 2a and $2 \mathrm{~b}$ show visual insight for LSI and its local Moran values respectively, with darkest shade corresponding to the highest quartile. These maps displayed geographical inequalities across governorates of Iraq. Based on visual inspection taken from Figure 2a, an overall worsening pattern (higher scores) was found in the southern part of the country. Suggestion of spatial clustering of similar values that follows a visual inspection of mapping was confirmed by a positive significant global Moran's $I$ of .34 with an associated $z$-statistic of 3.04 and $p=.002$.

To investigate global clustering, permutation test was done, where the permutation $p=.006$ was found significant. Thus, the null hypothesis of no spatial autocorrelation was rejected accordingly. Eight significant clusters were found in the western-northern and southern parts as shown in Figure 2b. These clusters: 2, 4, 5, 11, $12,14,15$ and 16 had higher level in deprivation compared to other governorates as shown from their local Moran values and their $p$-values in boldface in Table 2 .

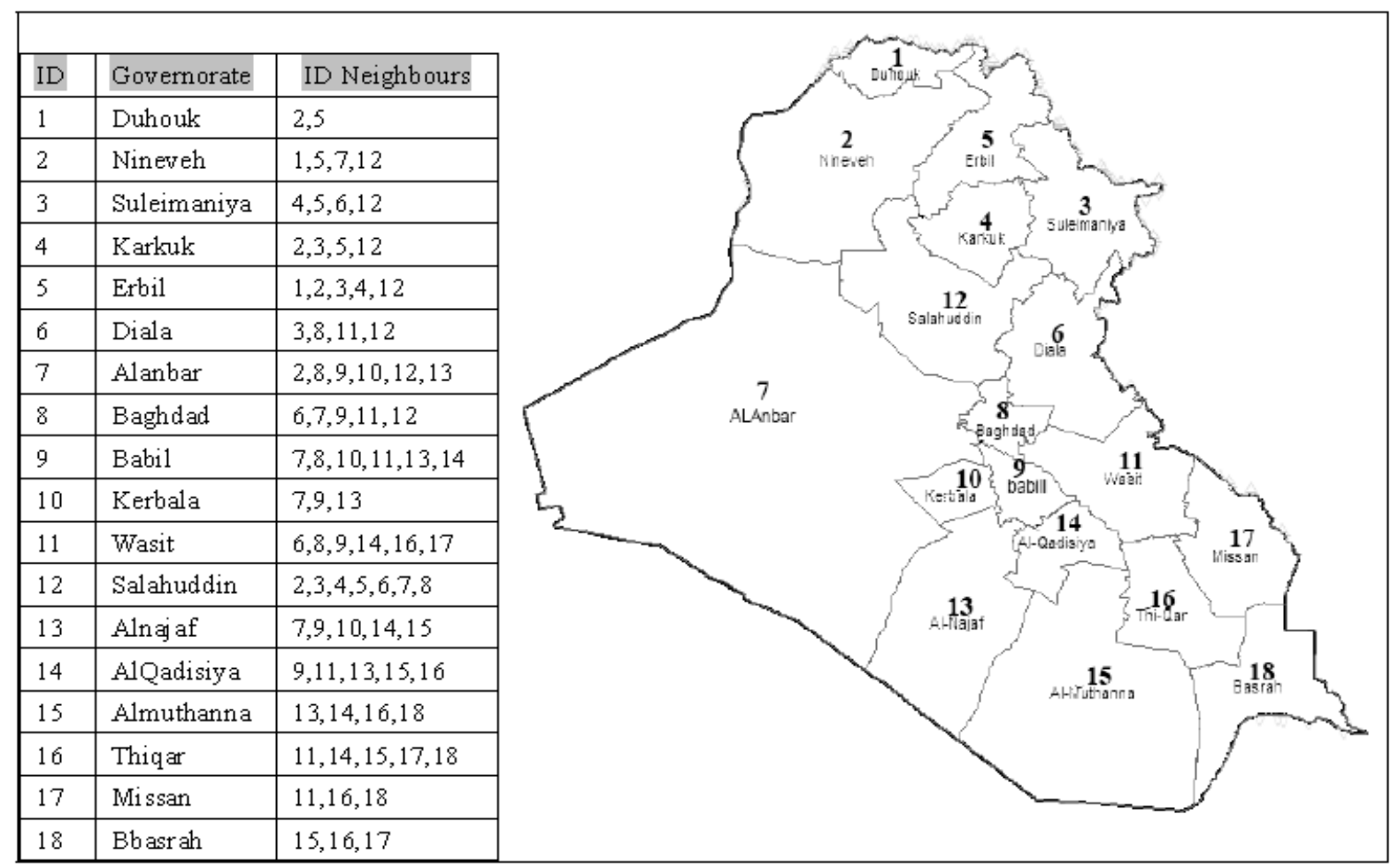

Figure 1. Study area shows all governorates with their ID and the neighbours of each governorate 


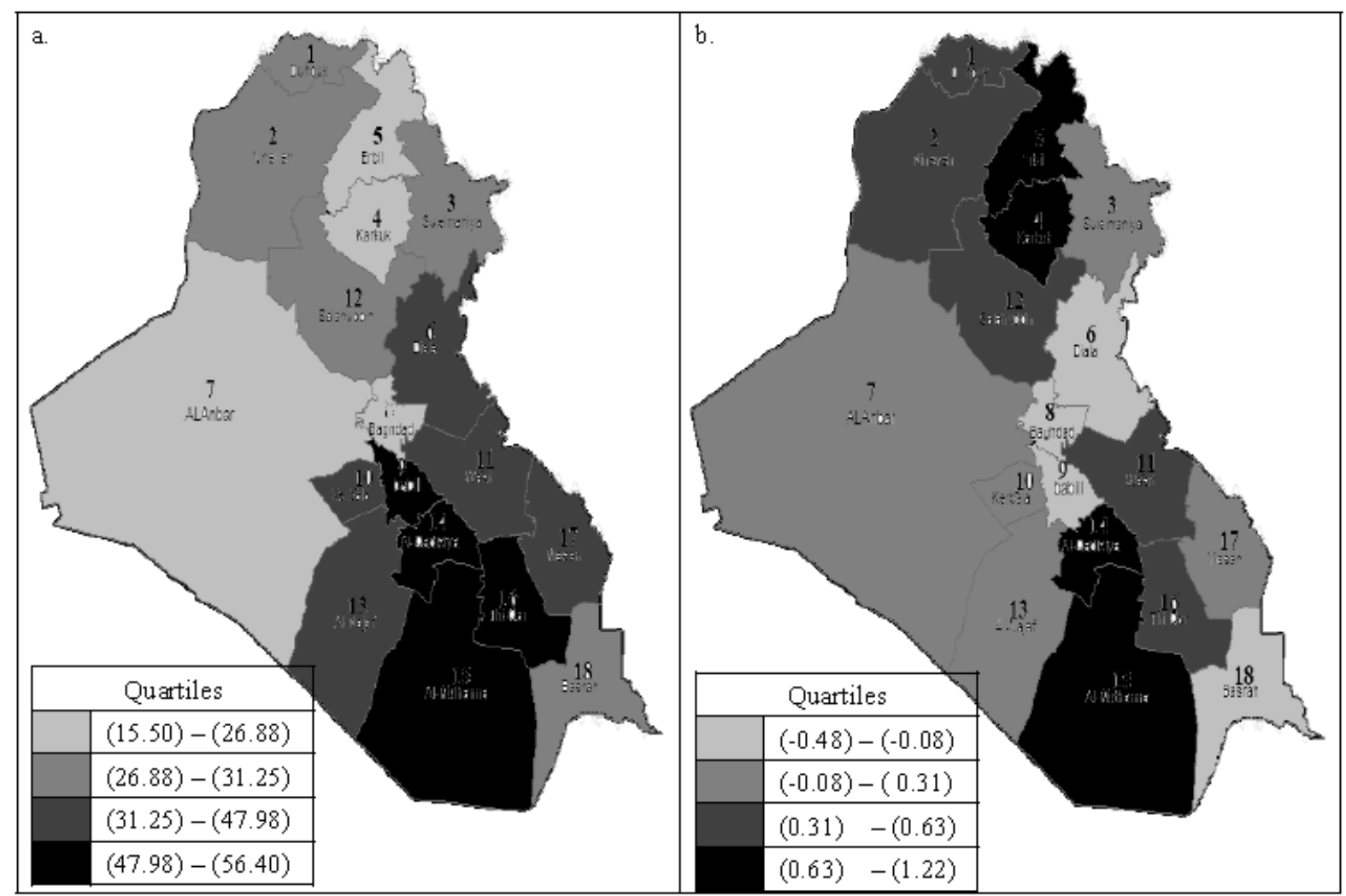

Figure 2. Choropleth maps show: a. LSI variable and b. its local Moran values

Table 2. Explains governorates, population size of each governorate, LSI, local Moran's $I_{i}$ and its corresponding $p$-value ( $p$-values in boldface were considered significant at .1 level)

\begin{tabular}{cccccc}
\hline ID & Governorate & Population size & LSI & $I_{i}$ & $p$ \\
\hline 1 & Duhouk & 472238 & 28.60 & .58 & .111 \\
2 & Nineveh & 2554270 & 29.00 & .48 & $\mathbf{. 0 7 3}$ \\
3 & Suleimaniya & 1715585 & 29.40 & .30 & .136 \\
4 & Karkuk & 854470 & 20.40 & 1.04 & $\mathbf{. 0 1 9}$ \\
5 & Erbil & 1392093 & 15.50 & 1.04 & $\mathbf{. 0 0 4}$ \\
6 & Diala & 1418455 & 47.40 & -.37 & .840 \\
7 & Alanbar & 1328776 & 22.90 & -.04 & .562 \\
8 & Baghdad & 6554126 & 20.40 & -.34 & .849 \\
9 & Babil & 1493718 & 55.50 & .16 & .171 \\
10 & Kerbala & 787072 & 45.60 & -.08 & .615 \\
11 & Wasit & 971280 & 43.50 & .33 & $\mathbf{. 0 7 2}$ \\
12 & Salahuddin & 1119369 & 28.50 & .41 &. $\mathbf{0 3 4}$ \\
13 & Alnajaf & 978400 & 38.80 & .19 & .169 \\
14 & AlQadisiya & 911641 & 51.60 & 1.22 & $\mathbf{. 0 0 1}$ \\
15 & Almuthanna & 554994 & 56.40 & .77 & $\mathbf{. 0 2 2}$ \\
16 & Thiqar & 1472405 & 49.70 & .56 & $\mathbf{. 0 3 4}$ \\
17 & Missan & 762872 & 33.10 & -.07 & .601 \\
18 & Albasrah & 1797821 & 28.20 & -.48 & .858
\end{tabular}

Figures $3 \mathrm{a}$ and $3 \mathrm{~b}$ show visual insight for education and its local Moran values respectively, with darkest shade 
corresponding to the highest quartile. Based on visual inspection on Figure 3a, an overall worsening pattern (higher scores) was found in the southern parts of the country. The suggestion of spatial clustering of similar values that follows a visual inspection of mapping was not confirmed by global Moran's $I$ of -.16 with an associated $z$-statistic of -.75 and $p=.451$. To investigate global clustering, permutation test was done, where permutation $p=.761$ was found not significant. Thus, the null hypothesis of no spatial autocorrelation was not rejected. One significant cluster was found in southern region as shown in Figure 2b, where its ID is 14 and has the worst level in education compared to other governorates as shown its local Moran value and its $p$-value in boldface in Table 3 .

Values of spatial measures indicate how much and to what extent, global clustering in studied variables is existed, and how many local clusters are there and where they are located. The z-statistic is the calculated value that is used to test the significance.

Figures $4 \mathrm{a}$ and $4 \mathrm{~b}$ show visual insight for HES and its local Moran values respectively, having darkest shade corresponding to the highest quartile. Based on visual inspection on Figure 4a, an overall worsening pattern (higher scores) was found in the southern part of the country. The suggestion of spatial clustering of similar values that follows a visual inspection of mapping was confirmed by a positive significant global Moran's $I$ of .28 having an associated $z$-statistic of 2.57 and $p=.010$. To investigate global clustering, permutation test was done, where permutation $p=.011$ was found significant. Thus, the null hypothesis of no spatial autocorrelation was rejected. Seven significant hot spots were found in the northern, middle, and western-southern parts as shown in Figure 4b, where their ID are: 4, 5, 6, 12, 13, 14, and 15 to have low level in HES compared to other governorates as shown in their local Moran values and their $p$-values in boldface in Table 3 .

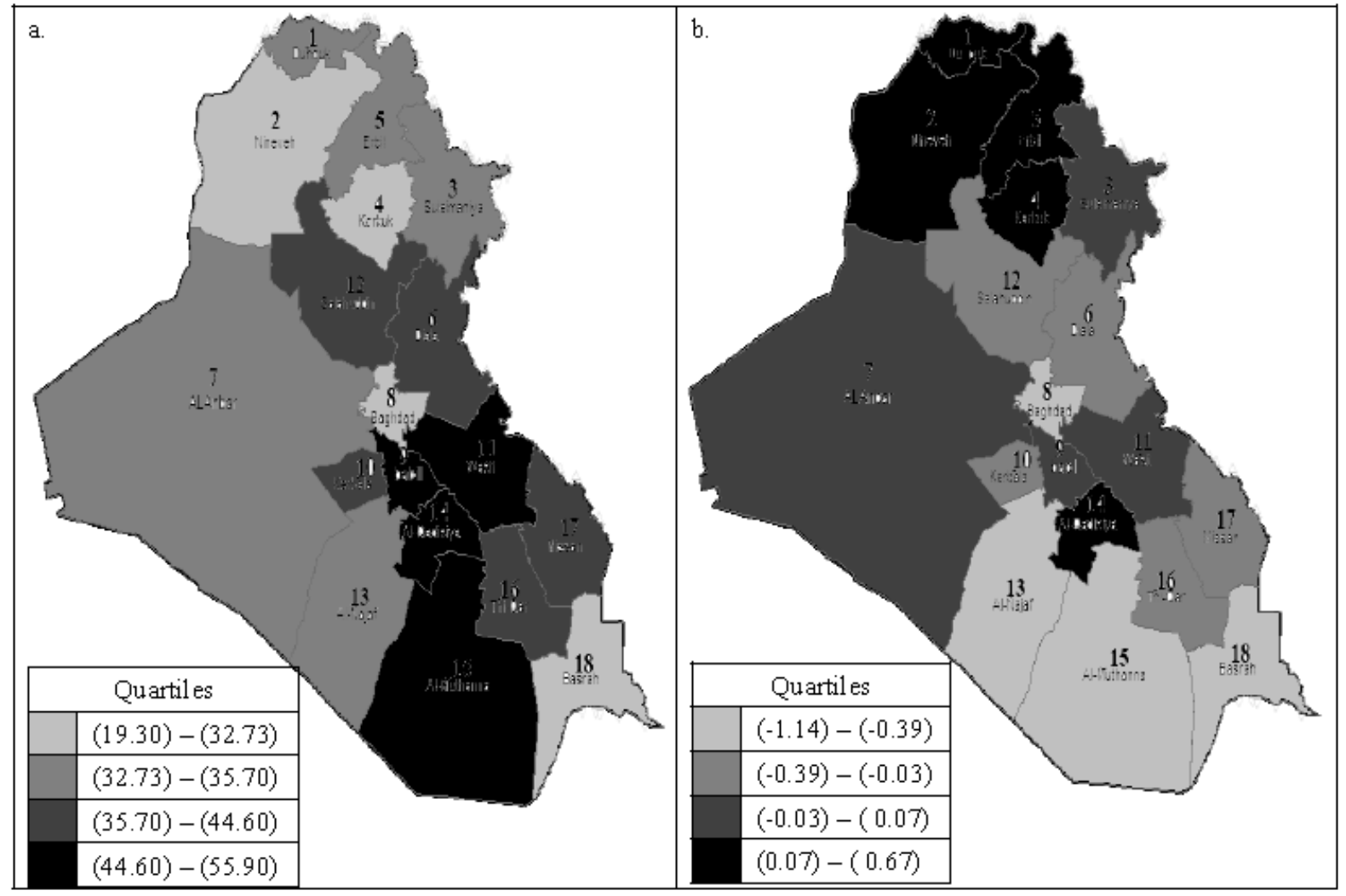

Figure 3. Choropleth maps show: a. education variable and $b$. its local Moran values 


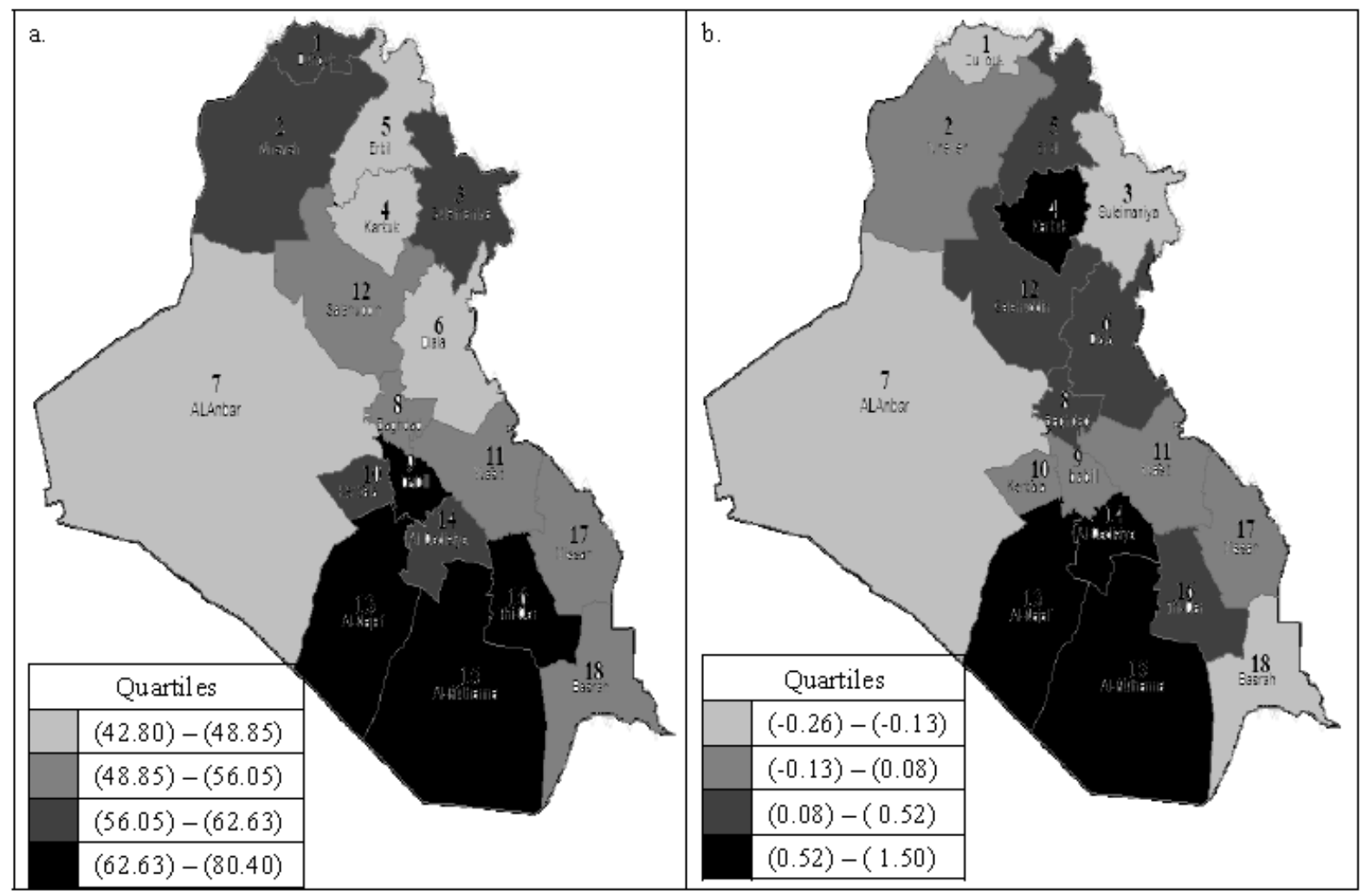

Figure 4. Choropleth maps show: a. HES variable and b. its local Moran values

Table 3. Explains governorates, education index, HES index, local Moran's $I_{i}$ for each index and its corresponding $p$-value ( $p$-values in boldface were considered significant at .1 level)

\begin{tabular}{cccccccc}
\hline ID & Governorate & Education & $I_{i}$ & $p$ & HES & $I_{i}$ & $p$ \\
\hline 1 & Duhouk & 35.6 & .08 & .355 & 58.90 & -.18 & .674 \\
2 & Nineveh & 32.2 & .07 & .325 & 57.20 & -.07 & .614 \\
3 & Suleimaniya & 35.1 & .03 & .365 & 57.90 & -.21 & .738 \\
4 & Karkuk & 29.4 & .07 & .339 & 42.80 & .92 & $\mathbf{. 0 2 6}$ \\
5 & Erbil & 34.6 & .09 & .267 & 43.90 & .43 & $\mathbf{. 0 6 2}$ \\
6 & Diala & 39.8 & -.07 & .606 & 47.10 & .38 & $\mathbf{. 0 9 8}$ \\
7 & Alanbar & 34.6 & .04 & .316 & 48.10 & -.19 & .767 \\
8 & Baghdad & 19.3 & -.99 & .979 & 54.20 & .11 & .240 \\
9 & Babil & 46.8 & -.02 & .505 & 63.80 & .02 & .377 \\
10 & Kerbala & 44.4 & -.36 & .835 & 58.10 & .04 & .352 \\
11 & Wasit & 45.9 & .05 & .310 & 49.10 & -.11 & .677 \\
12 & Salahuddin & 39.8 & -.17 & .763 & 49.50 & .48 & $\mathbf{. 0 2 1}$ \\
13 & Alnajaf & 32.9 & -.46 & .897 & 66.30 & .77 & $\mathbf{. 0 1 3}$ \\
14 & AlQadisiya & 45.2 & .67 & .021 & 62.60 & .63 & $\mathbf{. 0 2 4}$ \\
15 & Almuthanna & 55.9 & -.71 & .938 & 80.40 & 1.50 & $\mathbf{. 0 0 1}$ \\
16 & Thiqar & 35.8 & -.08 & .629 & 62.70 & .31 & .104 \\
17 & Missan & 38.5 & -.04 & .554 & 54.90 & .01 & .428 \\
18 & Albasrah & 23.3 & -1.14 & .969 & 54.00 & -.26 & .753 \\
\hline
\end{tabular}


Simulated data are useful for validating the results of spatial analysis. However, using Monte Carlo simulation, 9999 random samples (eighteen values for each sample) were simulated. The process of simulation was conducted under standard normal distribution to calculate $p$-values for local Moran values of LSI. When the word simulation is used, it is referred to an analytical method meant to imitate a real-life system, especially when other analyses are too mathematically complex or too difficult to reproduce. While results were specific to these data, the case study helps to identify general concepts for future study.

Living standards of the household are influenced by several indexes in various ways. However, spatial relationship between LSI and each of education and HES were investigated. Pearson correlation coefficient between LSI and education was found .76, which is significant with $p<.001$; and between LSI and HES was found .67 , which is significant with $p=.002$. The bivariate spatial correlation between LSI and education was found $\left(I_{x y}=.09\right)$, and it is not significant having $\mathrm{z}=.37$ and $p=.165$. Bivariate spatial correlation between LSI and HES was found $\left(I_{x y}=.31\right)$, and it is significant having $\mathrm{z}=1.28$ and $p=.001$. However, it is seen that Pearson coefficient is always over estimated when used in finding the spatial correlation. That's why, in investigating the bivariate spatial correlation, it is recommended to use Wartenberg (1985) measure.

\section{Discussion}

This study, undertaken in Iraq, quantified the spatial autocorrelation of HD. Spatial global clustering and local clusters for LSI were examined based on global Moran's $I$ and local Moran's $I_{i}$ respectively. Such findings allow policy makers to better identify what types of resources are needed and precisely where they should be employed. The above framework revealed some noteworthy findings. After rejecting the null hypothesis, it becomes possible to conclude that there was a form of global clustering and it was, of course, of interest to know the exact nature of this clustering. Are there hot-spot clusters? If so, how many hot-spots are there? and where are they located? However, the population size in these governorates is not equal; i.e. the rate of deprivation does not express the absolute size of the problem.

Exploratory tools such as descriptive table and somewhat small area choropleth maps were used. Maps provide powerful means to communicate data to others. Unlike information displayed in graphs, tables and charts; maps also provide bookmarks for memories. In this way, maps were not passive mechanism for presenting information. Usually, in the spatial analysis and geographical mapping, small spatial areas should be used such as districts, counties...etc. But, in this research governorates were used which considered somewhat larger than for example the districts because the data were not available for smaller areas. Most often the word 'neighbourhood' suggested a relatively of small area surrounding individuals' homes; but researchers commonly make use of larger spatial area such as census tracts (Coulton et al., 2001). Often, choices about neighbourhood spatial definitions were made with respect to convenience and availability of contextual data rather than study purpose (Schaefer-McDaniel et al., 2009). Schaefer-McDaniel et al. stated that, researchers might utilize census data and thus rely on census-imposed boundaries to define neighbourhoods even though these spatial areas may not be the best geographic units for the study topic. The spatial pattern showed the concentration of highly deprived areas in old areas such as AL-Muthana governorate and this deprivation is characterized by underprivileged living conditions. This was consistent with what found by Yuan, Fulong, and Xueqiang (2011) in the city of Guangzhou, China.

As noted by Waller and Jacques (1995), the test for spatial pattern employs alternative hypotheses of two types; the omnibus not the null hypothesis or more specific alternatives. Tests with specific alternatives include focused tests that are sensitive to monotonically decreasing risk as distance from a putative exposure source (the focus) increases. Acceptance of either types (the omnibus or a more specific alternatives) only demonstrates that some spatial pattern exist, and does not implicate a cause (Jacques, 2004). Hence, the existence of a spatial pattern alone cannot demonstrate nor prove a causal mechanism.

Anselin (1995) stated that indication of local pattern of spatial association may be in line with a global indication, although this is not necessarily be the case. It is quite possible that the local pattern is an aberration that the global indicator would not pick up, or it may be that a few local patterns run in the opposite direction of the global spatial trend. Local values that are very different from the mean (or median) would indicate locations that contribute more than their expected share to the global statistic. These may be outliers or high leverage points and thus would invite closer scouting. However, this is found in this research. Although global clustering in education was not found significant, one local cluster was found significant.

The usual correlation coefficients, such as Pearson coefficient, only test whether there is an association between two attributes by comparing values at the same location. While measuring spatial correlation using Wartenberg's measure and map comparison involve more than pair wise comparison between data recorded at the same 
locations as spatial units were arbitrary subdivisions of the study region and people could move around from one area to another. That could be affected by education and HES inequalities in areas other than the area they live in i.e., the inequalities of LSI in ith governorate were thought to be influenced and explained by the inequalities of education and HES not just in ith governorate but also in neighbouring governorates.

The application of statistical techniques to spatial data faces an important challenge, as expressed in the first law of geography: "everything is related to everything else, but closer things are more related than distant things" (Tobler, 1979). The quantitative expression of this principal is the effect of spatial dependence, i.e. when the observed values are spatially clustered, the samples are not independent. Deprivation growth in governorate $i$ generates deprivation growth in governorate $j$. This mechanism of transmission causes a spatial autocorrelation of deprivation growth. The obvious question after finding significant clusters in deprivation is-why? Could this pattern associated by the spatial pattern of socioeconomic indicators such as the levels of education or by the limitation of economic resources? However, further research regarding this bivariate spatial association between deprivation and socioeconomic indicators is required. It will be of an interest in the near future. This paper adds to the global body of knowledge on the utilization of spatial analysis to strengthen the research-policy interface in the developing countries. Although, this work was conducted as part of a wider study, its immediate implications are more for policy makers and practitioners than for researchers.

Maps displayed geographical inequalities in deprivation, education and HES across governorates of Iraq. Analytical approach used here delineates governorates of relatively high deprivation, and permits policy makers to develop strategies to minimize deprivation inequalities between governorates accurately. Policy which pays attention to area characteristics will reduce deprivation inequalities. Consequently improves the prosperity which in turn will improve health status.

This research was coming as continuing and as comprehensive study to other studies conducted in Iraq. It is worth to mention that there is high similarity between this study and other previous studies. For example, Khamis (2012a) investigated the spatial correlation of unemployment rate (UR) in Iraq in 2007. He found global clustering in UR and 7 governorates as local clusters in the eastern-northern and southern parts as found in this study. Also, Khamis and El-Refae (2012) studied the spatial patterns of acute malnutrition (AM) and household income (HI) in Iraq in 2004. They found global clustering for AM but they not found for HI. Out of 18 governorates, three and one governorates were found as local clusters in AM and HI respectively in central and western-southern parts. Bivariate spatial correlation between AM and HI was not found significant as in this study spatial correlation between LSI and education was not found significant. Khamis (2012c) examined the relationship between spatial patterns of chronic illnesses (CI) and UR in Iraq, 2007. He was not found significant spatial association between CI and UR. Also, HES was not found spatially associated with health in Iraq, 2004 by Khamis (2012b).

It should be emphasized that deprivation problem cannot be overcome in the short-run; but long-term efforts are needed to tackle the inequalities across governorates. In turn, enabling the economy to create more job opportunities and establish new projects, especially in the governorates that found as hot spot clusters. It means that the place of the problem is now clearly shown. Lack of investment in primary health care, primary education, and other life skills result in permanent life-long deprivation. Finally, deprivation studies should be conducted periodically in light of the changing of socioeconomic and political conditions.

Details presented in this research enables development actors to identify priorities, select fields and locate trends in designing national, regional, or sectorial policies. Moreover, this study provides civil society organizations, researches, and citizens with a rich knowledge base of facts and analysis, offering information easy to understand and to apply. This knowledge can be used either in targeted initiatives, or as a tool for monitoring and assessing policies and methods. Most importantly, the research provides the information needed to both examine development strategies' consistency with the actual situation, and to align priorities and develop them in accordance with the Iraqi people's development needs.

Although further work is required to gain a more complete understanding of the spatial clustering and of the spatial relationship between LSI and each of education and HES in Iraq, findings indicate that global clustering was existed in each of LSI and HES; but was not existed for education. Several governorates, as local clusters in LSI and in HES, were located in the northern and southern parts. Only one cluster in education was located in the southern part. However, spatial correlation between LSI and education was not existed as was not expected. But between LSI and HES was existed as was hypothesized by the researchers.

\section{Conclusions}

Conclusions are comprehensive in at least five aspects. First, visual inspection showed low level of LSI, 
education, and HES were concentrated in southern parts of the country. Second, several governorates were not observed visually as hot spots. But after considering the information of their neighbours, i.e. calculating their local Moran's $I_{i}$ values, the pattern of their hot spots can be obviously seen. Third, global clustering was found in each of LSI and HES, but was not found in education. Several governorates were found to be local clusters in LSI and in HES in northern and southern parts, and only one local cluster in education was found in southern part. The opposite was the case for those with high LSI, education, and HES were seen in central and some southern parts. Forth, from negative local Moran values, looking at local variation, some governorates represented as areas of dissimilarity in LSI. These governorates with low deprivation were surrounded by governorates with high deprivation or vice versa. Fifth, spatial correlation between LSI and education was not found significant but between LSI and HES was found significant. In summary, the study supports the hypothesis of a spatial inequality in deprivation at area level that probably reflects the inequality distribution in several socioeconomic indicators across governorates of Iraq.

Details presented enables development actors to identify priorities, select fields and locate trends in designing national, regional, or sectorial policies. Moreover, conclusions provide civil society organizations, researches, and citizens with a rich knowledge base of facts and analysis, offering information easy to understand and to apply. This knowledge can be used either in targeted initiatives, or as a tool for monitoring and assessing policies and methods.

\section{References}

Amerah, M. (1993). Unemployment in Jordan: dimensions and prospects. Center for international studies. Retrieved from http://library.fes.de/pdf-files/bueros/vifa-nahost/b93_00075.pdf

Anselin, L. (1995). Local Indicators of Spatial Association--LISA. Geographical Analysis, 27(2), 93-115. http://dx.doi.org/10.1111/j.1538-4632.1995.tb00338.x

Cliff, A. D., \& Ord, J. K. (1981). Spatial processes: Models \& Applications. London: Page Bros.

Coulton, C. J., Korbin, J., Chan, T., \& Su, M. (2001). Mapping residents' perceptions of neighbourhood boundaries: A methodological note. American journal of community psychology, 29(2), 371-383. http://dx.doi.org/10.1023/A:1010303419034

Gordon et al. (2007). Multidimensional Measures of Child Poverty.

Gordon, D., \& Spicker, P. (1999). The International Glossary on Poverty. ZED books: London/New York.

Haining, R. (2003). Spatial Data Analysis: Theory and Practice. Cambridge: Cambridge university press. http://dx.doi.org/10.1017/CBO9780511754944

Henninger, N. (1998). Mapping and geographic analysis of human welfare and poverty - review and assessment. World Resources Institute Washington, D.C., USA.

Hooghe, M., Vanhoutte, B., Hardyns, W., \& Bircan, T. (2011). Unemployment, Inequality, Poverty and Crime: Spatial Distribution Patterns of Criminal Acts in Belgium, 2001-06. British Journal of Criminology, 51(1), 1-20. http://dx.doi.org/10.1093/bjc/azq067

Hulme, D., Shepherd, A., \& Spray, P. (2006). The Chronic Poverty Report 2004-05. Retrieved from http://www.chronicpoverty.org/uploads/publication_files/CPR1_ReportFull.pdf

Jacques, G. M. (2004). Current practices in the spatial analysis of cancer: flies in the ointment. International journal of health geographics, 3(22), 1-10. Retrieved from http://www.ij-healthgeographics.com/content/3/1/22

Khamis, F. G. (2012a). Measuring the Spatial Correlation of Unemployment in Iraq-2007. Modern Applied Science, 6(1), 17-27.

Khamis, F. G. (2012b). The Relationship between Health and Household Economic Status Using Spatial Measures in Iraq, 2004. International Journal of Quality Statistics and Reliability, 1-8. http://dx.doi.org/10.1155/2012/481538

Khamis, F. G. (2012c). The Relationship between Spatial Patterns of Illnesses and Unemployment in Iraq-2007. Global Journal of Health Science, 4(1), 192-203.

Khamis, F. G., \& El-Refae, G. A. (2012). Association between Spatial Patterns of Acute Malnutrition and Household Income in Iraq-2004. International Journal of Statistics and Probability, 1(1), 43-52. http://dx.doi.org/10.5539/ijsp.v1n1p43 
Koch, T. (2005). Cartographies of disease: maps, mapping, and medicine (1st ed.). California: Guilford Press.

Lamb et al. (2010). Distribution of physical activity facilities in Scotland by small area measures of deprivation and urban city. International Journal of Behavioral Nutrition \& Physical Activity, 7, 76-83. http://dx.doi.org/10.1186/1479-5868-7-76

Lawson, A. B., \& Williams, F. R. (2001). An introductory guide to disease mapping. New York: Wiley \& Sons. http://dx.doi.org/10.1002/0470842571

Livingston et al. (2011). The patterning of deprivation and its effects on health outcomes in three postindustrial cities in Britain. Journal of Epidemiology Community Health, 65.

Macintyre, S., Maciver, S., \& Sooman, A. (1993). Area, class, and health: Should we be focusing on places or people? Journal of social policy, 22, 213-234. http://dx.doi.org/10.1017/S0047279400019310

Ministry of Planning and Development Cooperation/The Republic of Iraq. (2006). Unsatisfied basic needs mapping and living standards in Iraq. United Nations Development Program, 1-25.

Pozo et al. (2010). Chronic liver disease and cirrhosis mortality and social deprivation: a spatial analysis in small areas of Madrid region. Nutricion Hospitalaria, 25(4), 597-605.

Schabenberger, O., \& Gotway, C. A. (2005). Statistical methods for spatial data analysis. Boca Raton: Chapman \& Hall.

Schaefer-McDaniel, N., Caughy, M. O'B., O'Campo, P., \& Gearey, W. (2009) Examining methodological details of neighbourhood observations and the relationship to health: A literature review. Social science \& medicine, $1-16$.

Sridharan, S., Koschinsky, J., \& Walker, J. J. (2011). Does context matter for the relationship between deprivation and all-cause mortality? The West vs. the rest of Scotland. International Journal of Health Geographics, 10(1), 33-46. http://dx.doi.org/10.1186/1476-072X-10-33

Sven, V., Ursula, B., \& Razum, O. (2010).The impact of regional and neighbourhood deprivation on physical health in Germany: a multilevel study. Public Health, 10, 403-416.

Tobler, W. R. (1979). Cellular geography, In: Philosophy in Geography. Holland: DReidel Publishing Company. http://dx.doi.org/10.1007/978-94-009-9394-5_18

Townsend, P. (1987). Deprivation. Journal of Social Policy, 16(2), 125-146. http://dx.doi.org/10.1017/S0047279400020341

Tunstall et al. (2011). Socio-demographic diversity and unexplained variation in death rates between the most deprived areas in Britain. Journal of Epidemiology and Community Health, 65.

Waller, L. A., \& Gotway, C. A. (2004). Applied Spatial Statistics for Public Health Data. New Jersey: Wiley \& Sons. http://dx.doi.org/10.1002/0471662682

Waller, L. A., \& Jacques, G. M. (1995). Disease models implicit in statistical tests of disease clustering. Epidemiology, 6, 584-590. http://dx.doi.org/10.1097/00001648-199511000-00004

Wartenberg, D. (1985). Multivariate Spatial Correlation: A Method for Exploratory Geographical Analysis. Geographical Analysis, 17(4), 263-282. http://dx.doi.org/10.1111/j.1538-4632.1985.tb00849.x

Weeks, J. R. (1992). Population: An Introduction to concepts and Issues (5th. ed.). Wadsworth, Inc.

Yuan, Y., Fulong, W., \& Xueqiang, X. (2011). Multiple Deprivations in Transitional Chinese Cities: A Case Study of Guangzhou. Urban Affairs Review, 47(5), 721-736. http://dx.doi.org/10.1177/1078087411400370 